\title{
Corrigendum: Processing of visually evoked innate fear by a non-canonical thalamic pathway
}

Pengfei Wei, Nan Liu, Zhijian Zhang, Xuemei Liu, Yongqiang Tang, Xiaobin He, Bifeng Wu, Zheng Zhou, Yaohan Liu, Juan Li, Yi Zhang, Xuanyi Zhou, Lin Xu, Lin Chen, Guoqiang Bi, Xintian Hu, Fuqiang Xu \& Liping Wang

Nature Communications 6:6756 doi: 10.1038/ncomms7756 (2015); Published 9 Apr 2015; Updated 21 Aug 2015

The affiliation details for Lin $\mathrm{Xu}$ and Xintian $\mathrm{Hu}$ are incorrect in this article. The correct affiliation for these authors is given below. Kunming Institute of Zoology, CAS Center for Excellence in Brain Science, Chinese Academy of Sciences, Kunming 650223, China. article's Creative Commons license, unless indicated otherwise in the credit line; if the material is not included under the Creative Commons license, users will need to obtain permission from the license holder to reproduce the material. To view a copy of this license, visit http://creativecommons.org/licenses/by/4.0/
} 